Check for updates

Cite this: RSC Adv., 2018, 8, 28503

\title{
Gene function prediction based on combining gene ontology hierarchy with multi-instance multi-label learning
}

\author{
Zejun Li, ${ }^{\text {ab }}$ Bo Liao, (D) *a Yun Li, ${ }^{a}$ Wenhua Liu, ${ }^{b}$ Min Chen ${ }^{\text {ab }}$ and Lijun Cai ${ }^{a}$
}

Gene function annotation is the main challenge in the post genome era, which is an important part of the genome annotation. The sequencing of the human genome project produces a whole genome data, providing abundant biological information for the study of gene function annotation. However, to obtain useful knowledge from a large amount of data, a potential strategy is to apply machine learning methods to mine these data and predict gene function. In this study, we improved multi-instance hierarchical clustering by using gene ontology hierarchy to annotate gene function, which combines gene ontology hierarchy with multi-instance multi-label learning frame structure. Then, we used multi-label support vector machine (MLSVM) and multi-label k-nearest neighbor (MLKNN) algorithm to predict the function of gene. Finally, we verified our method in four yeast expression datasets. The performance of the simulated experiments proved that our method is efficient.

Received 14th June 2018

Accepted 12th July 2018

DOI: $10.1039 / \mathrm{c} 8 \mathrm{ra05122d}$

rsc.li/rsc-advances

expression profile across all areas of biology by integrating the annotations of the GO project. Huang et al. ${ }^{9}$ discussed various sorts and varieties of gene annotation enrichment analysis tools. Approximately 68 gene annotation enrichment tools that are currently available in the community were collected in this survey. These tools are uniquely categorized into three major classes, according to their underlying enrichment algorithms. Zhang $e t ~ a l{ }^{10}$ have created a web-based tool for data analysis and data visualization for sets of genes called GOTree Machine (GOTM). Although this tool was originally intended to analyze sets of co-regulated genes identified from microarray analysis, it is adaptable for use with other gene sets from other highthroughput analyses. Draghici et al. ${ }^{11}$ developed Onto-Express as a novel tool capable of automatically translating differentially regulated genes into functional profiles that characterize the impact of the condition studied. Despite the good performance of the machine learning techniques, there are still two characteristics of the function-prediction task that are different from common machine learning tasks: (1) a single gene may have multiple functions; and (2) the functions are organized in a hierarchy, i.e., a gene that is related to some functions is automatically related to all its ancestral functions (this is called the hierarchy constraint). ${ }^{12}$ Therefore, we combined multi-label learning frame with gene ontology hierarchy ${ }^{13}$ to settle this task.

In this study, we improved multi-instance hierarchical clustering (MIHC) ${ }^{14}$ with gene ontology hierarchy. Then, MLSVM and MLKNN classifiers were used to predict the function of genes in time-course gene expression profile. ${ }^{1}$ There are numerous classification methods that have been used in bioinformatics. ${ }^{15-18}$ In the section of Materials and methods, we will introduce the predicting task, MLSVM and MLKNN

${ }^{a}$ College of Information Science and Engineering, Hunan University, Changsha, Hunan, 410082, China

${ }^{b}$ School of Computer and Information Science, Hunan Institute of Technology, Hengyang, 412002, China 
algorithms. And, the MIHC method will be introduced. In the section of Experiment and results, we describe the application of MIHC method to the real data in GO database to examine its effectiveness. Numerical results show that the proposed method has better precision, recall-rate and harmonic mean value.

\section{Materials and methods}

\section{Gene function prediction task}

The goal of gene function annotation task is to find the function of un-annotation genes. The general calculation approach is to calculate the relationship between the genes and the various functions by a variety of biological models to predict the unannotation genes. ${ }^{19-23}$

From the correspondence between genes and its functions, a gene can be transcribed and translated into various proteins and can execute many different functions. ${ }^{24-27}$ Similarly, the in vivo biological process is not borne by a single gene, but by multiple genes working together. ${ }^{\mathbf{1 5}}$ Therefore, the relationship formed between the genes and its corresponding function is $\mathrm{N}$ to $\mathrm{N}$ mapping. Among the learning frameworks, the multiinstance multi-label learning framework is perfectly suited for $\mathrm{N}$ to $\mathrm{N}$ mapping. There is a certain degree of correlation between genes and genes and between gene functions and gene functions. However, the degradation processing in multiinstance multi-label learning framework destroys these correlations. Therefore, it is necessary to maintain the relevance when multi-instance multi-label learning framework is implemented.

\section{Gene ontology hierarchy}

GO database is a standard model with a hierarchical tree structure, designed to standardize biological knowledge of genes and their products. Overall, GO database is a directed acyclic graph (DAG), covering three branches: Biological Process (BP), Molecular Function (MF) and Cellular Components (CC). Also, there is no intersection between any two of the three branches. Moreover, GO database contains gene annotations of most of the microorganisms, plants and animal species, and GO terms can be used in multiple databases, maintaining the consistency of each gene described in different databases.

GO database was constructed by DAG, which treats GO terms as nodes of DAG and the relationship of GO terms as edges of DAG. The GO DAG also describes its terms by referring terms of the tree structure, such as tree root node, parent nodes, child nodes, leaf nodes and levels. This makes GO DAG easier to be understood. In GO DAG, the parent node closer to the root is described as rougher, while child nodes further from the root are described as finer. Therefore, genes annotated with the GO terms have the highest possible level of details, which corresponds to the lowest level of abstraction. ${ }^{28}$

There are three semantic relations between GO terms, namely, is_a, part_of and regulates. Among the three relationships, is_a and part_of relationships are transitive, while regulates relationships can be classified as the regulation of

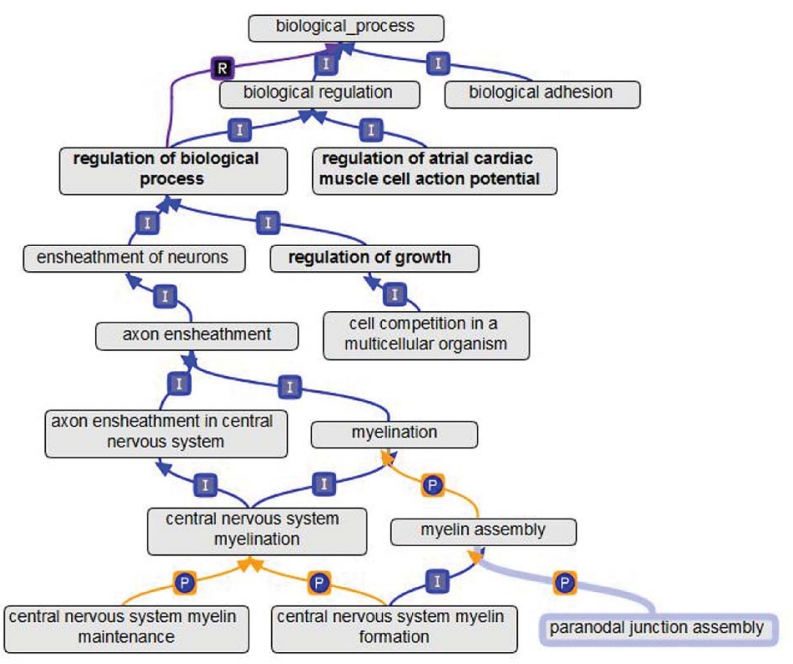

Fig. 1 Part of the gene ontology hierarchy relationships.

relations and under the control of the relationship. We simply describe these three relationships as follows. (1) The is_a relationship shows a relationship comprising a single included relationship. It also has the transitive. In other words, A is_a B represents $\mathrm{A}$ is subset of $\mathrm{B}$. Moreover, the relationship can be inferred from A is_a C and C is_a B. We formulated these derivation relationships as is_a $\times$ is_a $\rightarrow$ is_a. (2) The part_of relationships are similar to is_a relationships. A part_of $\mathrm{B}$ indicates that if $\mathrm{A}$ is present, then $\mathrm{A}$ is a subset of $\mathrm{B}$, but A does not necessarily occur. Similar to is_a relationship, part_of relationship also has the transitive. Therefore, we can also formulate the derivation relationships of part_of as part_of $\times$ part_of $\rightarrow$ part_of. (3) In comparison with the previous two relationships, the regulates relationship is slightly different. In GO semantics, if A can directly affect B, this affection, called A to $\mathrm{B}$, has a regulatory role, i.e., A regulates $\mathrm{B}$. The expressions of the three relationships in the GO database are shown in the Fig. 1. In Fig. 1, the alphabet "I" represents is_a relationship, "P" represents part_of relationship and " $R$ " represents regulates relationship.

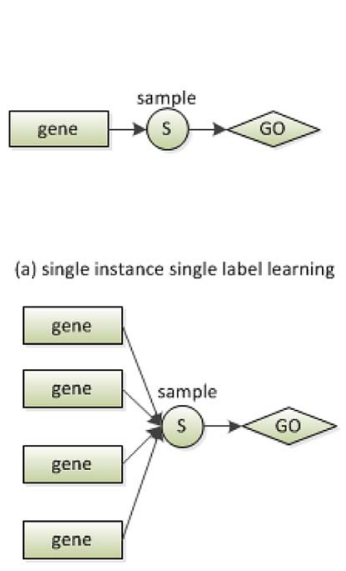

(c) Multi-instance learning

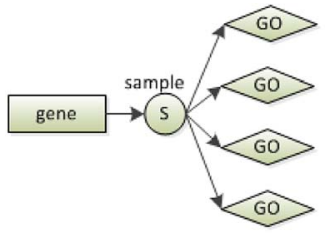

(b)Multi-label learning

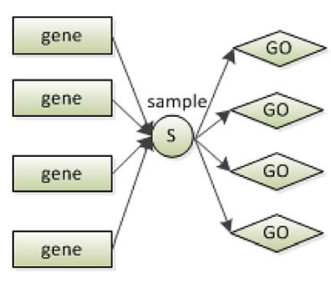

(d) Multi-instance learning

Fig. 2 Four types of machine learning frames. 
Table 1 The MLSVM algorithm

Input: $S$-the training set, T-the test samples

Output: $Y_{\mathrm{T}}$-the set of predicted labels of $\mathrm{T}$

(1)

Table 2 MLKNN algorithm
Input: $S$-the training set, T-the test samples Output: $Y_{\mathrm{T}}$-the set of predicted labels of $\mathrm{T}$

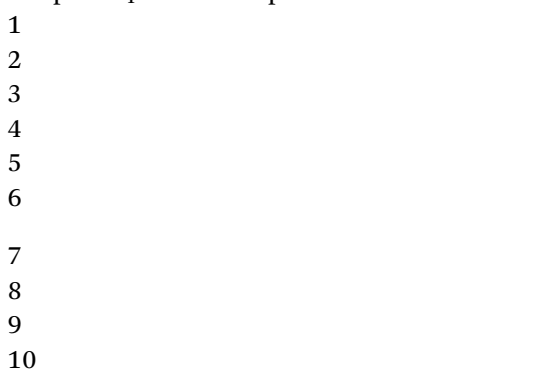

\section{MIML learning framework} follows:
For training set $S=\left\{\left(x_{i}, Y_{i}\right) \mid i=1,2, \ldots, N\right\}$, calculate the kernel matrix For each label $y \in Y, Y=\left\{Y_{i} \mid i=1,2, \ldots, N\right\}$

Produce a sub-training set $S_{y}=\left\{\left(x_{i}, \psi\left(x_{i}, y\right)\right) \mid i=1,2, \ldots, N\right\}$

Train a SVM model $M_{y}=\operatorname{svm} \operatorname{train}\left(S_{y}\right)$

For a test sample $t_{i} \in \mathrm{T}$

Its labels are obtained by $Y_{t i}=\left\{y \mid M_{y}\left(t_{i}\right) \geq 0\right\}$

End

End

$Y_{\mathrm{T}}=\left\{Y_{t i} \mid i=1,2, \ldots, N\right\}$
For a test sample $t_{i} \in \mathrm{T}$

Calculate $S_{t i} \in \mathrm{KNN}\left(t_{i}\right)$ which are the k-nearest neighbors of $t_{i}$ among the $S$

The candidate classes of $t_{i}$ are obtained by $Y_{t c}=\left\{y \mid y \in Y\right.$ and $\left.\psi\left(S_{t i}, Y\right)=1\right\}$

For each label $y \in Y_{t c}$

Calculate simScore $\left(t_{i}, s_{i}\right)$ which is the similarity score of $s_{i}$ to $t_{i}$

Calculate the likelihood score of $t_{i}$ to $y$ by Score $\left(t_{i}, y\right)=\sum_{s_{i} \in S_{t i}} \operatorname{simScore}\left(t_{i}, s_{i}\right) \psi\left(s_{i}, y\right)$

$t_{i}$ is labeled by $Y_{t i}=\left\{y \mid \operatorname{Score}\left(t_{i}, y\right) \geq 0\right\}$

End

End

$Y_{T}=\left\{Y_{t i} \mid i=1,2, \ldots, N\right\}$
The multi-instance multi-label learning (MIML) framework was proposed by Zhou et al. ${ }^{29}$ Formally, MIML can be defined as

Let $x=\left\{x_{1}, x_{2}, \ldots, x_{m}\right\}$ represent set of instances and $y=$ $\left\{y_{1}, y_{2}, \ldots, y_{n}\right\}$ denote set of labels. Given the dataset $\left\{\left(X_{1}, Y_{1}\right),\left(X_{2}, Y_{2}\right), \ldots,\left(X_{t}, Y_{t}\right)\right\}$, the goal of the learning task is the mapping of $f: 2^{x} \rightarrow 2^{y}$, where $X_{i} \subset x$ is a bag-of-instances while $Y_{i} \subset y$ is a subset of labels.

In this study, we solved the MIML task by degeneration approach. First, MIML task was degenerated into multi-
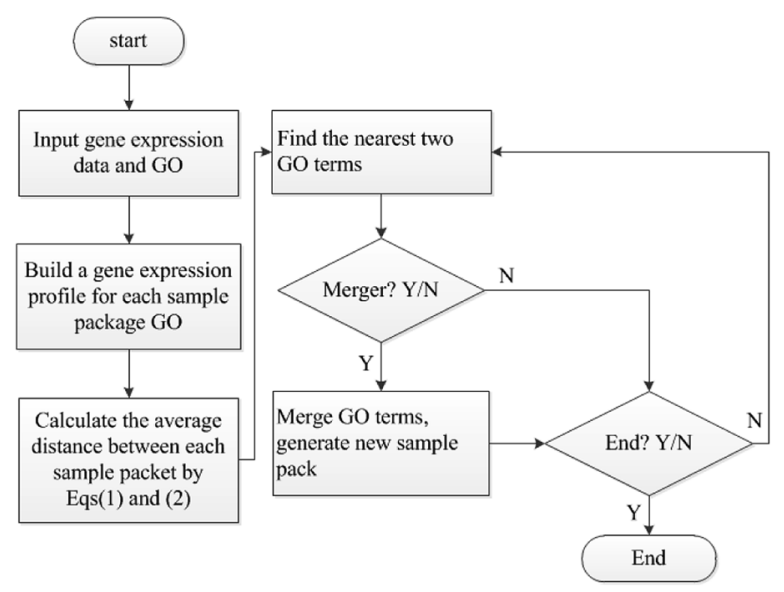

Fig. $3 \mathrm{MIHC}+$ algorithm flowchart. instance learning (MLL) or multi-label learning (MIL). Then, MLL or MIL was continually degenerated into single instance single label learning (SISL). The relationships of these learning frameworks are shown in Fig. 2. As shown in the subgraph (b), a gene is annotated by multiple GO terms. Fig. 2(c) shows that a GO term is a set of genes. The relationship shown in (b) is called multi-label, ${ }^{30}$ and that shown in (c) is called multiinstance. ${ }^{31}$ We used (b) or (c) as a bridge to degenerate (d) into (a). In this study, we used MLL algorithm to predict the annotation of novel genes. The pseudo code of MLL algorithms, which are MLSVM ${ }^{32,33}$ and MLKNN, ${ }^{34,35}$ are shown in Tables 1 and 2.

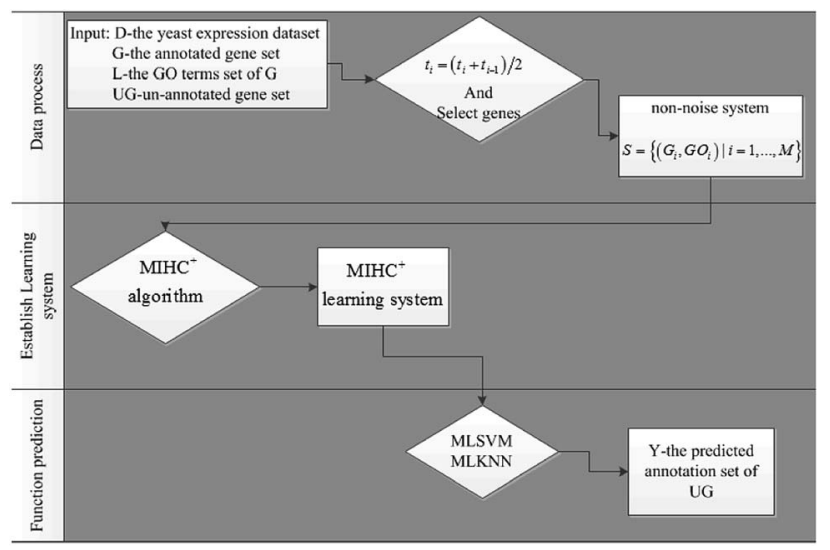

Fig. 4 The flow chart of gene function prediction. 


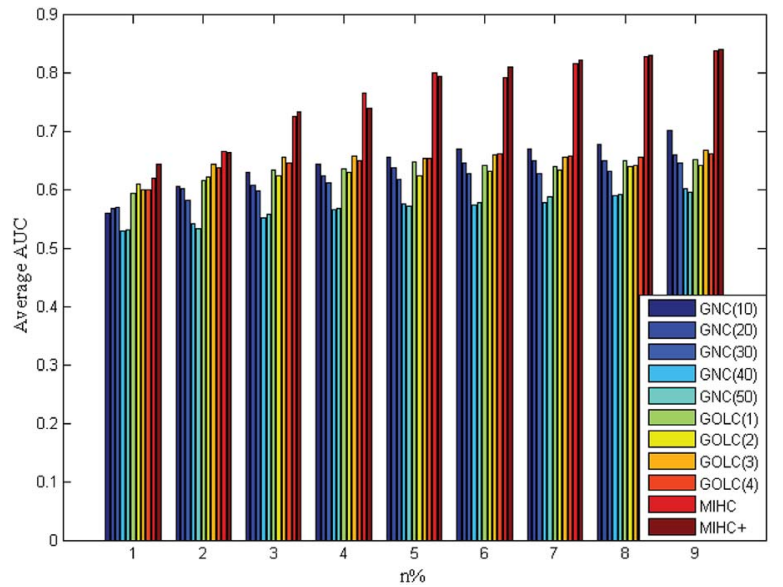

Fig. 5 The result obtained from each learning system by MLSVM in cdc28.

\section{MIHC+ algorithm}

Despite the MIHC algorithm making many efforts on gene annotation task, there are some limitations. MIHC does not consider the GO DAG when it clusters GO terms. In this study, we improved on this issue with GO hierarchy when GO terms were clustered.

Hierarchical Clustering ${ }^{36}$ is a widely used machine learning technology. General hierarchical clustering algorithm can be described as follows:

Step 1: determine all objects' dissimilarities by calculating the distance between each pair of objects, like Euclidean distance.

Step 2: collect two closest objects or clusters and merge them into one class.

Step 3: recalculate all dissimilarities between new clusters or objects.

Step 4: return to Step 2 until certain conditions are satisfied or certain number of clusters generated.

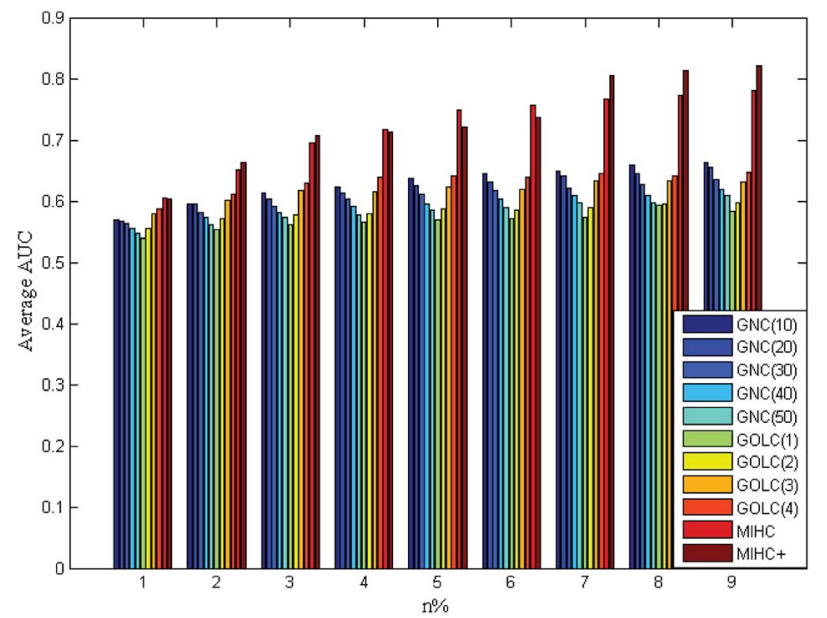

Fig. 6 The result obtained from each learning system by MLSVM in cdc15.

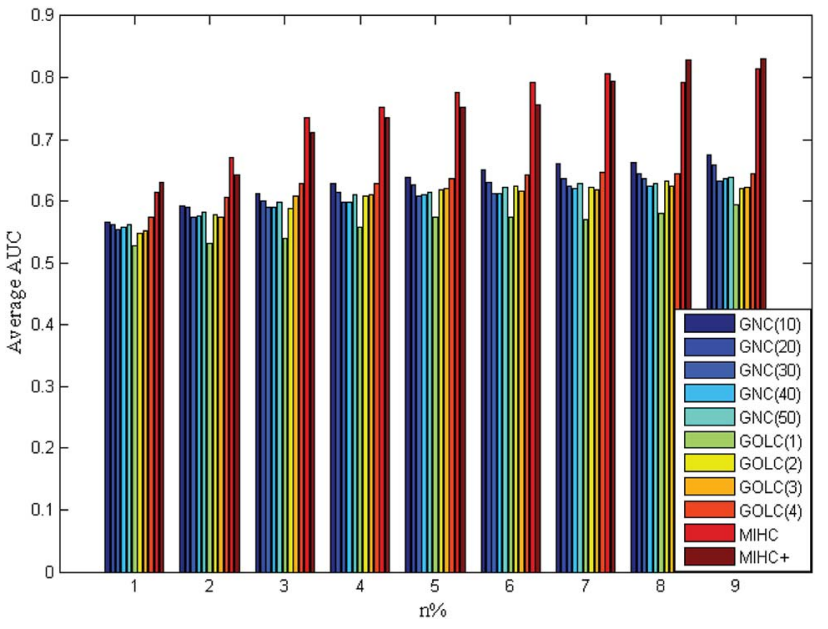

Fig. 7 The result obtained from each learning system by MLSVM in alph.

However, it is absurd that all objects are clustered into the same class. Hence, when certain conditions are satisfied, the algorithm is stopped. The end condition of MIHC algorithm is that no new cluster is generated. We still used formulae in MIHC to calculate the distance between bag-of-instances.

$$
\begin{gathered}
\operatorname{Corr}\left(\mathrm{G}_{i}\right)=\sum \mid \operatorname{corr}\left(\text { gene }_{i}, \text { gene }_{j}\right) \mid, \text { where gene } \\
\text {, } \text { gene }_{j} \in \mathrm{G}_{i} \\
D\left(\mathrm{GO}_{i}, \mathrm{GO}_{j}\right)=\overline{\operatorname{Corr}\left(G_{i} \cup G_{j}\right)}
\end{gathered}
$$

The flowchart of MIHC+ algorithm is shown in the Fig. 3. In the algorithm, the merger condition is the most important. We also defined the merger calculate condition as $D\left(\mathrm{GO}_{i}, \mathrm{GO}_{j}\right) \leq$ $\max \left(D\left(\mathrm{GO}_{i}\right), D\left(\mathrm{GO}_{j}\right)\right)$, but MIHC+ algorithm needs another condition to merge two GO terms. Following the GO hierarchy, we up-propagated the two GO terms; if one of them owns a common ancestor in the GO database, we merge them. If there are no more new GO terms needed to be merged, the algorithm comes to an end. In other words, MIHC+ algorithm completely obeys the knowledge of GO hierarchy.

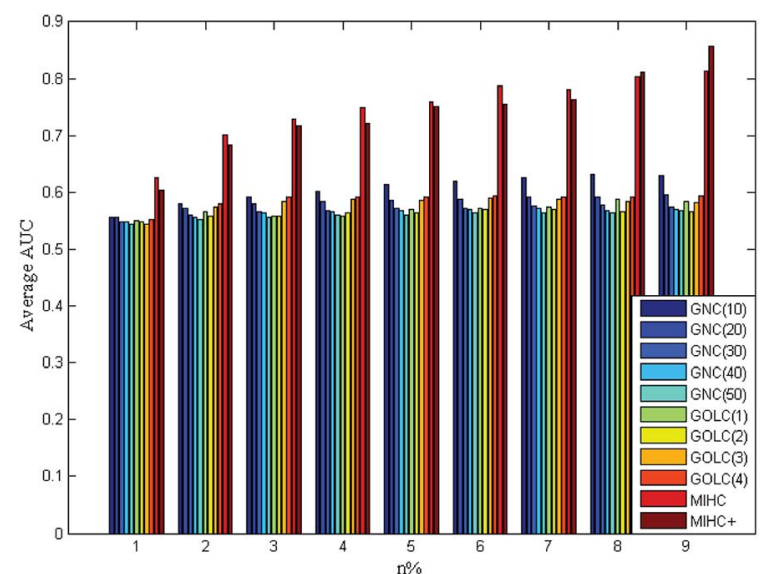

Fig. 8 The result obtained from each learning system by MLSVM in elution. 
Table 3 The results of cdc28 dataset by MLSVM

\begin{tabular}{|c|c|c|c|c|c|c|c|c|c|c|}
\hline \multirow[b]{2}{*}{ Method } & & \multicolumn{9}{|l|}{$n \%$} \\
\hline & & 10 & 20 & 30 & 40 & 50 & 60 & 70 & 80 & 90 \\
\hline \multirow[t]{5}{*}{ GNC } & $\lambda=10$ & 0.559 & 0.606 & 0.631 & 0.644 & 0.656 & 0.671 & 0.670 & 0.679 & 0.703 \\
\hline & $\lambda=20$ & 0.569 & 0.603 & 0.607 & 0.625 & 0.638 & 0.646 & 0.650 & 0.650 & 0.661 \\
\hline & $\lambda=30$ & 0.571 & 0.583 & 0.599 & 0.612 & 0.618 & 0.629 & 0.628 & 0.632 & 0.646 \\
\hline & $\lambda=40$ & 0.529 & 0.543 & 0.552 & 0.567 & 0.577 & 0.574 & 0.578 & 0.589 & 0.602 \\
\hline & $\lambda=50$ & 0.532 & 0.535 & 0.558 & 0.569 & 0.572 & 0.579 & 0.588 & 0.592 & 0.596 \\
\hline \multirow[t]{4}{*}{ GOLC } & $\iota=1$ & 0.594 & 0.617 & 0.634 & 0.635 & 0.648 & 0.643 & 0.641 & 0.651 & 0.653 \\
\hline & $\iota=2$ & 0.609 & 0.623 & 0.624 & 0.631 & 0.624 & 0.632 & 0.635 & 0.640 & 0.643 \\
\hline & $\iota=3$ & 0.601 & 0.644 & 0.657 & 0.658 & 0.654 & 0.661 & 0.656 & 0.643 & 0.668 \\
\hline & $\iota=4$ & 0.601 & 0.638 & 0.647 & 0.651 & 0.654 & 0.663 & 0.658 & 0.656 & 0.662 \\
\hline MIHC & & 0.621 & 0.666 & 0.727 & 0.767 & 0.800 & 0.794 & 0.817 & 0.828 & 0.838 \\
\hline $\mathrm{MIHC}^{+}$ & & 0.644 & 0.665 & 0.735 & 0.740 & 0.796 & 0.811 & 0.822 & 0.831 & 0.840 \\
\hline
\end{tabular}

\section{Experiment and results}

\section{Experiment}

Time-series expression datasets in the experiment were obtained from ref. 37, and can be downloaded from ref. 38. These four datasets are yeast cell cycle expression data with different time points and circumstances. Gene annotation data can be obtained from GO database, which can be downloaded from ref. 39 . We used the method in ref. 40 to preprocess the raw data and always make the first value 0 . Then, the average transformation $t_{i}=\left(t_{i}+t_{i-1}\right) / 2$ was used to smooth out spikes. After the data process, we used the method in ref. 14 to select genes that are significantly correlated with each other in the same function. Then, the non-noise system of expression data and annotation are represented as $S=\left\{\left(\mathrm{G}_{i}, \mathrm{GO}_{i}\right) \mid i=1, \ldots, M\right\}$. Subsequently, the MIHC+ algorithm was used to the construct learning system. Finally, MLSVM and MLKNN classifiers were used to verify the performance of the learning system. The flow chart of the gene function prediction is shown in Fig. 4.

Leave-one-out and leave-a-percent-out cross validation ${ }^{41}$ approaches were used for evaluating the performance of the function prediction algorithm. We selected the latter method to evaluate the MIHC+ method. To accurately measure the performance, the receiver operating characteristic (ROC) curve and area under the ROC curve (AUC) were introduced to quantify the results. The classifications were often based on continuous random variables. The probability of belonging in a class varies with different threshold parameters. In other words, the values of true and false positive rates (TPR and FPR, respectively) vary with different threshold parameters. The ROC curve parametrically plots TPR versus FPR with varying parameters. TPR and FPR were calculated by eqn (3) and (4).

Table 4 Some GO terms and its genes in alph dataset

\begin{tabular}{lllll}
\hline Environment & Alph & & & \\
Genes & YBR189W & YGL189C & YGR214W & YJR123W \\
& YER025W & YGR094W & YGR285C & YNL178W \\
YGL123W & YGR118W & YHR064C & YNL209W \\
GO terms & GO:0008152 & GO:0009987 & GO:0044237 & GO:0044238
\end{tabular}

Table 5 Some GO terms and its genes in cdc15 datasets

\begin{tabular}{|c|c|c|c|c|c|}
\hline Environment & cdc15 & & & & \\
\hline \multirow[t]{6}{*}{ Genes } & YBR048W & YGL030W & YKL006W & YLR333C & YOL120C \\
\hline & YDL061C & YGL103W & YKR057W & YLR367W & YOL127W \\
\hline & YDL083C & YGR034W & YKR094C & YLR388W & YOR063W \\
\hline & YDR418W & YHR203C & YLR167W & YML091C & YPL131W \\
\hline & YER102W & YIL069C & YLR185W & YNL209W & \\
\hline & YFR031C-A & YIL133C & YLR264W & YOL040C & \\
\hline & GO:0000478 & GO:0006807 & GO:0030490 & GO:0044085 & GO:0090304 \\
\hline & GO:0000479 & GO:0008152 & GO:0034470 & GO:0044237 & GO:0090305 \\
\hline & GO:0000480 & GO:0009987 & GO:0034641 & GO:0044238 & GO:0090501 \\
\hline & GO:0006139 & GO:0010467 & GO:0034660 & GO:0044260 & GO:0090502 \\
\hline & GO:0006364 & GO:0016070 & GO:0042254 & GO:0046483 & GO:1901360 \\
\hline
\end{tabular}


Table 6 Some GO terms and its genes in cdc28 datasets

\begin{tabular}{|c|c|c|c|c|c|}
\hline Environment & $\operatorname{cdc} 28$ & & & & \\
\hline \multirow[t]{6}{*}{ Genes } & YBL027W & YDL191W & YJR145C & YLR367W & YOR312C \\
\hline & YBL087C & YDR025W & YKL180W & YNL162W & YPL143W \\
\hline & YBR048W & YDR064W & YKR057W & YNL302C & YPL198W \\
\hline & YBR084C-A & YDR447C & YKR094C & YOL120C & YPR132W \\
\hline & YBR181C & YHL001W & YLR185W & YOL121C & \\
\hline & YDL075W & YJL189W & YLR287C-A & YOR234C & \\
\hline GO terms & GO:0009987 & GO:0044699 & GO:0044763 & & \\
\hline
\end{tabular}

Table 7 Some GO terms and its genes in cdc28 datasets

\begin{tabular}{|c|c|c|c|c|c|}
\hline Environment & Elution & & & & \\
\hline \multirow[t]{8}{*}{ Genes } & YBL047C & YEL048C & YJL154C & YLR361C & YNL192W \\
\hline & YBL099W & YER096W & YJR017C & YLR371W & YOR273C \\
\hline & YBR038W & YFL038C & YJR032W & YLR417W & YOR332W \\
\hline & YBR127C & YFR026C & YJR121W & YML034W & YPR156C \\
\hline & YCR069W & YGR106C & YKL002W & YML078W & YPR165W \\
\hline & YDL089W & YGR138C & YKL080W & YMR054W & \\
\hline & YDR304C & YHL006C & YKL203C & YMR089C & \\
\hline & YDR519W & YHR079C & YLR106C & YNL026W & \\
\hline GO terms & GO:0009987 & & & & \\
\hline
\end{tabular}

$$
\begin{aligned}
& \mathrm{TPR}=\mathrm{TP} /(\mathrm{TP}+\mathrm{FN}) \\
& \mathrm{FPR}=\mathrm{FP} /(\mathrm{FP}+\mathrm{TN})
\end{aligned}
$$

where TP, FP, TN and FN represent the number of true positive, false positive, true negative and false negative predictions, respectively. Therefore, TPR and FPR can reflect the sensitivity and specificity of prediction. AUC was calculated to quantify the content of the ROC curves. A reliable and valid AUC estimate can be interpreted as the probability that the classifier will assign a higher score to a randomly chosen positive sample rather than to a randomly chosen negative sample.

\section{Results}

The four yeast time-course expression datasets are alpha, cdc15, cdc28 and elution, which record mRNA level of 18, 24, 17 and 14 time points in whole cell cycle under different circumstances, respectively. For each expression dataset, MIHC+ and other three methods (GNC, GOLC and MIHC) in ref. 14 were used to construct learning system. Then, all learning systems were tested by MLSVM and MLKNN classifiers. In the classification task, the multi-label learning task is decomposed into a series of binary classification tasks. The experimental settings are the same as that in ref. 14. For each expression dataset, the average results obtained from each learning system by MLSVM classifier are shown in Fig. 5-8. The data in these figures indicate that the MIHC+ learning system has a similar performance with MIHC. The results from cdc28 dataset are shown in Table 3. However, MIHC+ method can give more biological information. From MIHC+ learning system, we found that the GO term named 'GO: 0009987' appears in all of these datasets and only 7 genes, which own 'GO: 0009987', appear in cdc28 and cdc15 dataset. From the GO, we find that 'GO: 0009987' named "cellular process" is defined as "any process that is carried out at the cellular level, but not necessarily restricted to a single cell". For example, cell communication occurs among more than one cell, but at the cellular level.

The results of the experiments in four datasets proved that genes involved in the same biological processes may vary with external environment. Moreover, the ref. 37 also points towards this view because yeast cells automatically turn on or turn off certain genes' expression in order to adapt to the external environment when cells are in different growth environments. We present some GO terms and its genes for four different datasets in Table 4-7. As summarized in these tables, all of genes in the unit of "Genes" have the corresponding GO terms in the unit of "GO terms".

\section{Conclusion}

In this study, we improve the MIHC method with gene ontology hierarchy (MIHC+ method) to construct a learning system. Our method was verified on four yeast gene expression datasets. The MIHC+ method treats gene ontology hierarchy as the relationship between gene annotations and then, Hierarchical Clustering follows the GO hierarchy to cluster them. Compared with other learning systems employed in this study, the MIHC+ method obtained more biological knowledge from the timeseries expression dataset. It also has a similar performance with MIHC method. In future research, we will combine gene annotation information with other biological information (e.g., single nucleotide polymorphism, ${ }^{\mathbf{4 2 , 4 3}}$ and miRNA $^{\mathbf{4 4 - 5 2}}$ ) to diagnose complex diseases more accurately.

\section{Conflicts of interest}

There are no conflicts to declare. 


\section{Acknowledgements}

This study was supported by National Natural Science Foundation of China (Grant Nos. 61672223, 61672214 and 61772192), National Nature Science Foundation of Hunan Province (Grant No. 2016JJ4029). Key research and development project of Hunan Province (Grant No. 2015GK3029).

\section{References}

1 X. Chen, Y. A. Huang, Z. H. You, G. Y. Yan and X. S. Wang, Bioinformatics, 2016, 33, 733-739.

2 B. Liao, Y. Jiang, G. Yuan, W. Zhu, L. Cai and Z. Cao, PLoS One, 2014, 9, e104314.

3 X. Zhou, M. C. J. Kao and W. H. Wong, Proc. Natl. Acad. Sci. U. S. A., 2002, 99, 12783-12788.

4 X. M. Zhao, Y. Wang, L. Chen and K. Aihara, BMC Bioinf., 2008, 9, 1-14.

5 Z. Barutcuoglu, R. E. Schapire and O. G. Troyanskaya, Bioinformatics, 2006, 22, 830-836.

6 A. Vinayagam, R. König, J. Moormann, F. Schubert, R. Eils, K. H. Glatting and S. Suhai, BMC Bioinf., 2004, 5, 116.

7 Z. L. Pei, X. H. Shi, M. Niu, X. N. Tang and L. S. Liu, J. Bionic Eng., 2007, 4, 177-184.

8 S. W. Doniger, N. Salomonis, K. D. Dahlquist, K. Vranizan, S. C. Lawlor and B. R. Conklin, Genome Biol., 2003, 4, R7.

9 D. W. Huang, B. T. Sherman and R. A. Lempicki, Nucleic Acids Res., 2009, 37, 1.

10 B. Zhang, D. Schmoyer, S. Kirov and J. Snoddy, BMC Bioinf., 2004, 5, 1-8.

11 S. Draghici, P. Khatri, R. P. Martins, G. C. Ostermeier and S. A. Krawetz, Genomics, 2003, 81, 98-104.

12 L. Schietgat, C. Vens, J. Struyf, H. Blockeel, D. Kocev and S. Dzeroski, BMC Bioinf., 2010, 11, 2.

13 B. Liao, X. Li, L. Cai, Z. Cao and H. Chen, IEEE/ACM Trans. Comput. Biol. Bioinf., 2015, 12, 113-122.

14 B. Liao, Y. Li, Y. Jiang and L. Cai, PLoS One, 2014, 9, e90962.

15 X. Chen and L. Huang, PLoS Comput. Biol., 2017, 13, e1005912.

16 C. Xing, B. Ren, C. Ming, Q. Wang, L. Zhang and G. Yan, PLoS Comput. Biol., 2016, 12, e1004975.

17 B. Liao, X. Li, W. Zhu and Z. Cao, IEEE/ACM Trans. Comput. Biol. Bioinf., 2012, 9, 1529-1534.

18 B. Liao, B. Liao, X. Sun and Q. Zeng, Bioinformatics, 2010, 26, 2678-2683.

19 X. Chen, D. Xie, Q. Zhao and Z. H. You, Briefings Bioinf., 2017, 18, 558.

20 X. Chen, C. C. Yan, X. Zhang and Z.-H. You, Briefings Bioinf., 2016, bbw060.

21 B. Liao, Y. Jiang, W. Liang, W. Zhu, L. Cai and Z. Cao, IEEE/ ACM Trans. Comput. Biol. Bioinf., 2014, 11, 1146-1156.

22 Y. Wang, Z. You, L. Xiao, C. Xing, T. Jiang and J. Zhang, Int. J. Mol. Sci., 2017, 18, 1029.

23 Z. H. You, Z. A. Huang, Z. Zhu, G. Y. Yan, Z. W. Li, Z. Wen and X. Chen, PLoS Comput. Biol., 2017, 13, e1005455.
24 X. Chen, C. C. Yan, X. Zhang, X. Zhang, F. Dai, J. Yin and Y. Zhang, Briefings Bioinf., 2016, 17, 696.

25 X. Chen and G. Y. Yan, Bioinformatics, 2013, 29, 2617-2624. 26 X. Li, B. Liao, L. Cai, Z. Cao and W. Zhu, IEEE/ACM Trans. Comput. Biol. Bioinf., 2013, 10, 688-695.

27 Z. W. Li, Z. H. You, X. Chen, J. Gui and R. Nie, Int. J. Mol. Sci., 2016, 17, 1396.

28 P. Khatri and S. Drăghici, Bioinformatics, 2005, 21, 35873595.

29 Z. H. Zhou, M. L. Zhang, S. J. Huang and Y. F. Li, Corros. Abstr., 2008, arxiv: abs/0808.3231.

30 G. Tsoumakas, I. Katakis and D. Taniar, Int. J. Data Warehous. Min., 2007, 3, 1-13.

31 Z. H. Zhou, Multi-instance learning: A survey, Technical Report, 2004,Department of Computer Science \& Technology, 2004, p. 1-31.

32 Y. X. Li, S. Ji, S. Kumar, J. Ye and Z. H. Zhou, IEEE/ACM Trans. Comput. Biol. Bioinf., 2012, 9, 98-112.

33 C. Cortes and V. Vapnik, Mach. Learn., 1995, 20, 273-297.

34 T. Denœux, IEEE Trans. Syst. Man Cybern. Syst., 2008, 25, 804-813.

35 M. L. Zhang and Z. H. Zhou, Pattern Recognit., 2007, 40, 2038-2048.

36 S. C. Johnson, Psychometrika, 1967, 32, 241-254.

37 P. T. Spellman, G. Sherlock, M. Q. Zhang, V. R. Iyer, K. Anders, M. B. Eisen, P. O. Brown, D. Botstein and B. Futcher, Mol. Biol. Cell, 1998, 9, 3273-3297.

38 http://genome-www.stanford.edu/cellcycle/data/rawdata/.

39 http://www.geneontology.org/

GO.downloads.annotations.shtml.

40 J. Ernst, G. J. Nau and Z. Barjoseph, Bioinformatics, 2005, 1(21 Suppl), i159.

41 A. Magi, L. Tattini, M. Benelli, B. Giusti, R. Abbate and S. Ruffo, PLoS One, 2012, 7, e38767.

42 X. Li, Bioinformatics, 2017, 33, 2829-2836.

43 X. Li, Curr. Bioinf., 1969, 12, 367-372.

44 X. Chen, C. C. Yan, X. Zhang, Z. H. You, L. Deng, Y. Liu, Y. Zhang and Q. Dai, Sci. Rep., 2016, 6, 21106.

45 G. Li, J. Luo, X. Qiu, L. Cheng and P. Ding, J. Biomed. Inf., 2018, 82, 169-177.

46 G. Li, J. Luo, Q. Xiao, C. Liang, P. Ding and B. Cao, IEEE Access, 2017, 5, 24032-24039.

47 G. Li, J. Luo, Q. Xiao, C. Liang and P. Ding, RSC Adv., 2018, 8, 4377-4385.

48 X. Chen, L. Wang, J. Qu, N.-N. Guan and J.-Q. Li, Bioinformatics, 2018, DOI: 10.1093/bioinformatics/bty503.

49 X. Chen, D. Xie, L. Wang, Q. Zhao, Z. H. You and H. Liu, Bioinformatics, 2018.

50 H. Lee, S. Lee and H. L. Chang, Appl. Math. Comput., 2016, 286, 232-249.

51 X. Chen, L. Huang, D. Xie and Q. Zhao, Cell Death Dis., 2018, 9, 3.

52 J. Qu, X. Chen, Y. Z. Sun, J. Q. Li and Z. Ming, J. Cheminf., 2018, 10, 30 . 